\title{
Research Article \\ Strength Mechanism and Influence Factors for Cold Recycled Asphalt Mixture
}

\author{
Tao Ma, ${ }^{1,2}$ Hao Wang, ${ }^{2}$ Yongli Zhao, ${ }^{1}$ Xiaoming Huang, ${ }^{1}$ and Yuhui Pi \\ ${ }^{1}$ School of Transportation, Southeast University, Nanjing 210096, China \\ ${ }^{2}$ Department of Civil and Environmental Engineering, Rutgers, The State University of New Jersey, Piscataway, NJ 08854, USA \\ Correspondence should be addressed to Tao Ma; matao@seu.edu.cn
}

Received 9 July 2015; Accepted 6 September 2015

Academic Editor: Akihiko Kimura

Copyright (c) 2015 Tao Ma et al. This is an open access article distributed under the Creative Commons Attribution License, which permits unrestricted use, distribution, and reproduction in any medium, provided the original work is properly cited.

\begin{abstract}
This study focused on the key factors affecting the tensile strength of cold recycled asphalt mixture with cement and emulsified asphalt. The specific surface areas and strength of RAP were analyzed. The interaction between the emulsified asphalt and cement was observed. Comprehensive laboratory testing was conducted to evaluate the influences of RAP, emulsified asphalt, and cement on the tensile strength of cold recycled asphalt mixture. It is found that although RAP is used as aggregates, its inner structure and strength are much different from real aggregates. The strength of RAP has decisive effect on the strength of cold recycled asphalt mixture. New aggregates and fine gradation design can help improve the bonding between RAP and binder. For emulsified asphalt, slow setting of asphalt can give sufficient time for cement to hydrate which is helpful for strength formation in the cold recycled asphalt mixture. The high viscosity of asphalt can improve the early strength of cold recycled asphalt mixture that is important for traffic opening in the field. Cement is an efficient additive to improve the strength of cold recycled asphalt mixtures by promoting demulsification of emulsified asphalt and producing cement hydrates. However, the cement content is limited by RAP.
\end{abstract}

\section{Introduction}

Recycling technology has become one of the most promising approaches for asphalt pavement rehabilitation and maintenance with considerable savings in natural resource, cost, and energy [1-3]. It has been proved that hot recycled asphalt mixture with controlled RAP content between $10 \%$ and $30 \%$ performs similarly to virgin hot-mix asphalt mixture [4]. However, with the continuously increasing energy costs and environmental awareness, central-plant hot recycling technologies have difficulties meeting the need of using high-content RAP in asphalt pavements. Compared to hot recycling technology, cold recycling technology can use much higher RAP contents and bring more benefits $[5,6]$. Therefore, cold recycling technology has attracted significant attentions in recent years.

The overall process of cold recycling includes milling, processing, mix design, binder addition, placement, compaction, and quality assurance [7]. To successfully use cold recycling for asphalt pavements, a lot of researches have been conducted on the mix design method and evaluation of mechanical properties of cold recycled asphalt mixtures. Based on previous research findings [8-13], the "black rock" theory is the basis for current design of cold recycled asphalt mixtures. The asphalt binder in RAP is usually ignored and RAP plays a single role of aggregate. Emulsified asphalt or foamed asphalt is used as new binder in cold recycled asphalt mixtures. However, it was found that the cold recycled asphalt mixture that is composed of RAP and emulsified or foamed asphalt may not perform well to carry heavy traffic loading. Thus, additives such as Portland cement, lime, and fly ash are suggested to be used to improve the performance of cold recycled asphalt mixture. Among these additives, adding Portland cement is an efficient way to consume water in the emulsified or foamed asphalt and form a stronger and denser skeleton in the cold recycled asphalt mixture [14]. In this case, the cold recycled asphalt mixture is regarded as semiflexible material different from the normal asphalt mixture and cement concrete $[15,16]$.

Studies have shown that many agencies used cold recycled asphalt mixtures in the base layer. However, some problems with low early strength, extended curing time, cracking, and 
TABLE 1: Gradations for coarse, median, and fine RAP.

\begin{tabular}{|c|c|c|c|c|c|c|c|c|c|c|c|c|c|}
\hline \multirow{2}{*}{ RAP } & \multicolumn{13}{|c|}{ Passing ratios/\% for different sieving sizes $/ \mathrm{mm}$} \\
\hline & 31.5 & 26.5 & 19 & 16 & 13.2 & 9.5 & 4.75 & 2.36 & 1.18 & 0.6 & 0.3 & 0.15 & 0.075 \\
\hline Coarse & 100 & 77.9 & 38.0 & 0 & & & & & & & & & \\
\hline Median & & 100 & 100 & 100 & 90.4 & 69.2 & 29.1 & 0.0 & & & & & \\
\hline Fine & & 100 & 100 & 100 & 100 & 100 & 100 & 100 & 53 & 29 & 8.4 & 3.6 & 0.6 \\
\hline
\end{tabular}

raveling were commonly observed from field experience [1720]. One of the most important reasons for causing these problems is the strength forming mechanism in the cold recycled asphalt mixture. Although RAP may look like "black" aggregate, the contribution of RAP to the cold recycled asphalt mixture may not be the same as real aggregate due to the existence of aged asphalt binder. If RAP does not play the same role as real aggregate, the mix design that is based on "black rock" for RAP may lead to improper mixture design and poor pavement performance. As Portland cement is added to improve strength of the cold recycled asphalt mixture, the cement hydration could be affected by the existence of asphalt binder in RAP and the emulsified or foamed asphalt. Meanwhile, it is not clear if the aggregate gradation and the type or content of asphalt emulsion have significant influences on the performance of cold recycled asphalt mixtures.

The main objective of this study is to understand the strength forming mechanism and its key influence factors for the cold recycled asphalt mixture with cement and emulsified asphalt. To analyze the strength mechanism of cold recycled asphalt mixture, the inner structure and strength between RAP and real aggregate were compared and the interaction between the emulsified asphalt and cement was investigated. The influences of RAP, emulsified asphalt, and cement on the tensile strength of cold recycled asphalt mixture were evaluated.

\section{Materials and Methods}

2.1. Materials. RAP was obtained from milling materials of asphalt layers of an expressway which had been on service for 8 years. To control the variability of gradation design for the cold recycled asphalt mixture, RAP was crushed and then sieved into three grades which are coarse RAP bigger than $16 \mathrm{~mm}$, median RAP between $16 \mathrm{~mm}$ and $2.36 \mathrm{~mm}$, and fine RAP smaller than $2.36 \mathrm{~mm}$. The RAP particles for which the size is bigger than $30 \mathrm{~mm}$ were excluded for use. The gradations of coarse RAP, median RAP, and fine RAP are shown in Table 1.

New fine aggregates and mineral fillers with qualities meeting the requirement by Chinese Technical Specification for Construction of Highway Asphalt Pavement [21] were used to improve the gradation design. The gradations of fine aggregates and mineral fillers are shown in Table 2.

Emulsified asphalt, cement, and potable water were used as binder in the cold recycled asphalt mixture. Three different types of emulsified asphalt, normal slow-setting emulsified asphalt, normal medium-setting emulsified asphalt, and high-viscosity slow-setting emulsified asphalt, were used in
TABLE 2: Gradations for new aggregates and mineral fillers.

\begin{tabular}{lcccccccc}
\hline \multirow{2}{*}{ Material } & \multicolumn{1}{c}{ Passing ratios/\% for different sieving sizes $/ \mathrm{mm}$} \\
& 9.5 & 4.75 & 2.36 & 1.18 & 0.6 & 0.3 & 0.15 & 0.075 \\
\hline New aggregates & 100 & 99.5 & 75.7 & 55.5 & 35.7 & 26.5 & 13.8 & 4.5 \\
Mineral fillers & & & & & & 100 & 90 & 80 \\
\hline
\end{tabular}

this study. Table 3 shows the basic rheological properties of the emulsified asphalt. Ordinary Portland cement with grade 32.5 was used and its physical properties are shown in Table 4. All three types of emulsified asphalt and the Portland cement meet the requirement by Chinese Technical Specification for Construction of Highway Asphalt Pavement [21].

One controlling cold recycled mixture was prepared following Chinese Technical Specification for Highway Asphalt Pavement Recycling [22]. The aggregate mix of cold recycled asphalt mixture includes 75\% RAP, 20\% new aggregates, and $5 \%$ mineral fillers for gradation. The new binder added to the mixture was composed of $4 \%$ emulsified asphalt, $1.5 \%$ cement, and $5.4 \%$ water by the weight of the aggregate mix. Based on the mix design of the controlling cold recycled asphalt mixture, different gradations, emulsified asphalt types and contents, and cement contents were used to prepare cold recycled asphalt mixtures for analysis.

2.2. Laboratory Testing. Specific surface area is defined as the total surface area per unit mass of material. The specific surface area of aggregates has significant influence on the binder content and the adhesion between binder and aggregate. Specific surface area can be calculated using the gradation and the specific area coefficient for different sieving sizes, as shown in (1). The specific surface area coefficients $\left(F A_{i}\right)$ for different sieving sizes of aggregates are shown in Table 5 . The specific surface area of both RAP and the aggregates extracted from RAP was analyzed in this study:

$$
\mathrm{SA}=\sum P_{i} \times F A_{i}
$$

where SA is the total specific surface area of different sieving sizes, $\mathrm{m}^{2} / \mathrm{kg} ; P_{i}$ is the passing ratio of different sieving sizes, $\%$; and $F A_{i}$ is the specific surface area coefficient for different sieving sizes.

Scanning electron microscope (SEM) can be used to observe the microstructure of material by scanning the material with electron and analyzing the secondary electron promoted on the surface of the material. Cement and emulsified asphalt were mixed based on quality ratio of $1: 1$ and then cured for 7 days at ambient temperature to form the cementasphalt mix. Then asphalt was solved and eliminated from 
TABLE 3: Basic properties of emulsified asphalt.

\begin{tabular}{|c|c|c|c|c|}
\hline \multirow{2}{*}{ Indexes } & \multicolumn{3}{|c|}{ Emulsified asphalt } & \multirow{2}{*}{ Specification } \\
\hline & High-viscosity & Slow-setting & Medium-setting & \\
\hline Demulsification speed & Slow & Slow & Medium & Slow or medium \\
\hline Particle charge & $(+)$ & $(+)$ & $(+)$ & $(+)$ \\
\hline Residual stay on $1.18 \mathrm{~mm} / \%$ & 0.05 & 0.04 & 0.08 & $\leq 0.1$ \\
\hline Englar viscosity & 22 & 9 & 10 & $2-30$ \\
\hline \multicolumn{5}{|l|}{ Residual after distillation } \\
\hline Content $/ \%$ & 66 & 60 & 62 & $\geq 55$ \\
\hline Penetration $\left(25^{\circ} \mathrm{C}\right) / 0.1 \mathrm{~mm}$ & 55 & 69 & 72 & $50-300$ \\
\hline Ductility $\left(15^{\circ} \mathrm{C}\right) / \mathrm{cm}$ & 60 & 110 & 95 & $\geq 40$ \\
\hline Solubility $/ \%$ & 98.5 & 98.1 & 99.8 & $\geq 97.5$ \\
\hline
\end{tabular}

the cement-asphalt mix to obtain the cement hydrates. In this study, SEM was used to observe the microstructures of both the cement-asphalt mix and the cement hydrates extracted from the cement-asphalt mix.

The tensile strength is the most important mechanical property for design of cold recycled asphalt mixtures. Splitting test can be used to determine the tensile strength of mixtures at biaxial stress states. Samples with diameter of $101.6 \mathrm{~mm}$ and height of $63.5 \mathrm{~mm}$ were prepared. Splitting test was conducted with a loading rate of $50 \mathrm{~mm} / \mathrm{min}$ at $15^{\circ} \mathrm{C}$. The tensile strength of samples was calculated based on (2). There are two different testing conditions that can be used for splitting test. One is called dry splitting test in which the testing specimen is conditioned at $15^{\circ} \mathrm{C}$ in the air bath for $6 \mathrm{~h}$ or in the water bath for $2 \mathrm{~h}$ before splitting test. The other one is called immersion splitting test in which the testing specimen is conditioned at $25^{\circ} \mathrm{C}$ in the water bath for $22 \mathrm{~h}$ and then at $15^{\circ} \mathrm{C}$ in the water bath for another $2 \mathrm{~h}$ before splitting test. The tensile strength by the splitting test can be determined by (2). And the tensile strength ratio defined by (3) can be used to evaluate the moisture stability of test specimen:

$$
R_{T}=\frac{0.006287 P_{T}}{h}
$$

where $R_{T}$ is the splitting tensile strength, $\mathrm{MPa} ; P_{T}$ is the maximum loading when sample is split, $\mathrm{MPa}$; and $h$ is the sample height, $\mathrm{mm}$. Consider

$$
\mathrm{TSR}=\frac{R_{2}}{R_{1}} \times 100,
$$

where TSR is the tensile strength ratio, $\% ; R_{2}$ is the tensile strength from immersion splitting test, $\mathrm{MPa}$; and $R_{1}$ is the tensile strength from dry splitting test, MPa.

\section{Results and Discussion}

\subsection{Effect of RAP}

3.1.1. Structure and Strength Analysis of RAP. Compared to real aggregates, RAP material may have similar shapes and sizes. However, many coarse particles in RAP are actually
TABLE 4: Basic properties of cement.

\begin{tabular}{lcc}
\hline Indexes & Testing value & Specification \\
\hline Initial setting time/min & 147 & $\geq 45$ \\
Final setting time/min & 326 & $\leq 600$ \\
Compressive strength/MPa & & \\
$\quad 3 \mathrm{~d}$ & 13.2 & $\geq 11.0$ \\
$\quad 28 \mathrm{~d}$ & 33.8 & $\geq 32.5$ \\
Flexural strength/MPa & & \\
$\quad 3 \mathrm{~d}$ & 3.9 & $\geq 2.5$ \\
$28 \mathrm{~d}$ & 6.2 & $\geq 5.5$ \\
\hline
\end{tabular}

TABLE 5: Specific surface area coefficients for different sieving sizes of aggregates.

\begin{tabular}{lccccccc}
\hline Sieving size $(i) / \mathrm{mm}$ & $\geq 4.75$ & 2.36 & 1.18 & 0.6 & 0.3 & 0.15 & 0.075 \\
\hline$F A_{i}\left(10^{-2}\right) / \mathrm{m}^{2} / \mathrm{kg}$ & 0.4 & 0.8 & 1.64 & 2.87 & 6.14 & 12.3 & 32.77 \\
\hline
\end{tabular}

agglomerates of fine RAP particles bonded by the aged asphalt. This can be proved by the significant gradation difference between RAP and the extracted aggregates from RAP, as shown in Figure 1. It clearly shows that the gradation of extracted aggregates from RAP is much finer than the gradation of RAP. The biggest particle size of the extracted aggregates is $19 \mathrm{~mm}$ while it is $26.5 \mathrm{~mm}$ for RAP. In addition, there are much fewer RAP particles passing through the sieve of $0.3 \mathrm{~mm}$ compared to the extracted aggregates from RAP. To quantify the influence of agglomeration in RAP, the specific surface areas of RAP and the extracted aggregates from RAP were calculated using (1). The results are shown in Figure 2.

From Figure 2, it can be seen that the specific surface area of the extracted aggregates is about three times the specific surface area of RAP. It indicates that when the new binder is added into RAP, only about one-third of the total surface of the aggregates in RAP can be covered and bonded by the new binder. The remaining surface of the aggregates in RAP is still covered by the aged asphalt in RAP. This explains why the asphalt content of cold recycled asphalt mixture is usually lower than hot-mix asphalt mixture. More importantly, the adhesion strength of aged asphalt binder inside RAP is much weaker than new binder due to long-term aging. Thus, when 


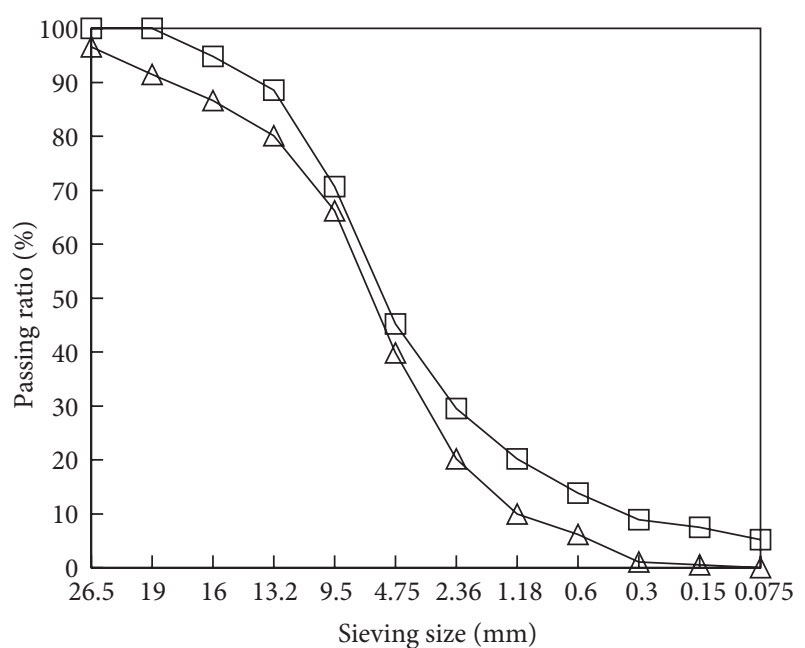

$\triangle$ RAP

$\square$ Extracted aggregate

Figure 1: Gradations of RAP and aggregates.

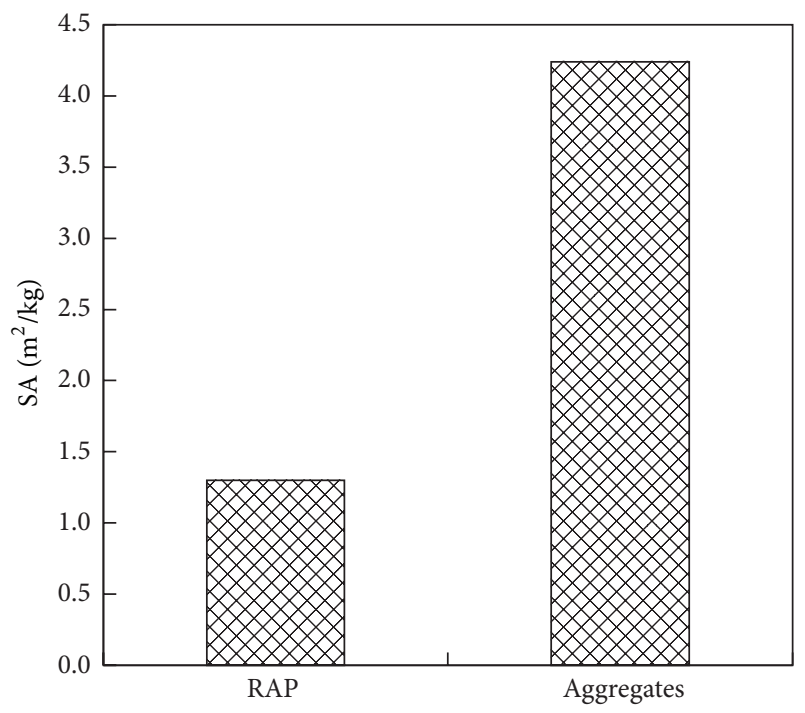

FIgURE 2: SA of RAP and aggregates.

subjected to mechanical and environmental loading, the internal surface inside the RAP particles can be weak spots in the cold recycled asphalt mixture.

Splitting test was conducted to estimate the tensile strength of RAP. Since the splitting test cannot be used directly to determine the tensile strength of RAP, testing samples were prepared by mixing RAP with cement mortar. Cement mortar was prepared with water, cement, and sand according to a mass ratio of $1: 2: 3$. Testing samples were prepared by mixing cement mortar and RAP at different RAP contents $(0,10,20,30,40$, and $50 \%$ by the weight of the total mix). The relationship between the tensile strength of cement-mortar RAP samples and the RAP content is summarized in Figure 3.

It can be seen that the tensile strength decreases as the percentage of RAP increases. A linear relationship was

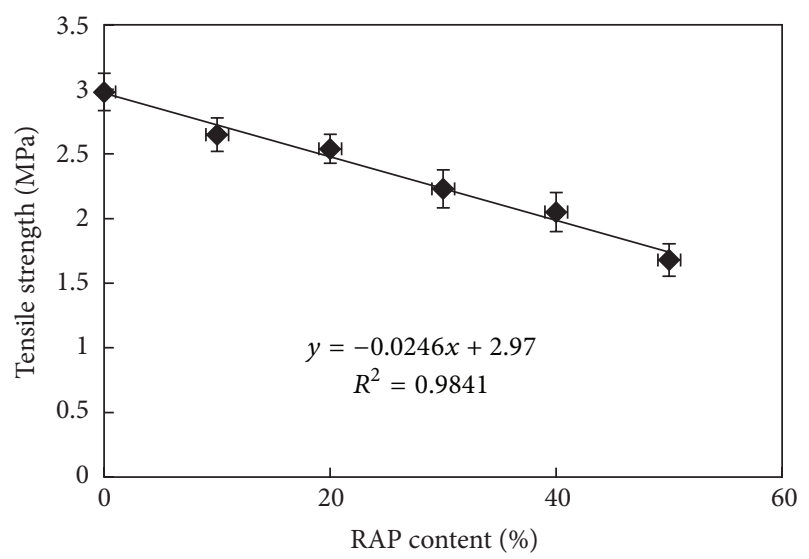

FIGURE 3: Tensile strength versus RAP content.

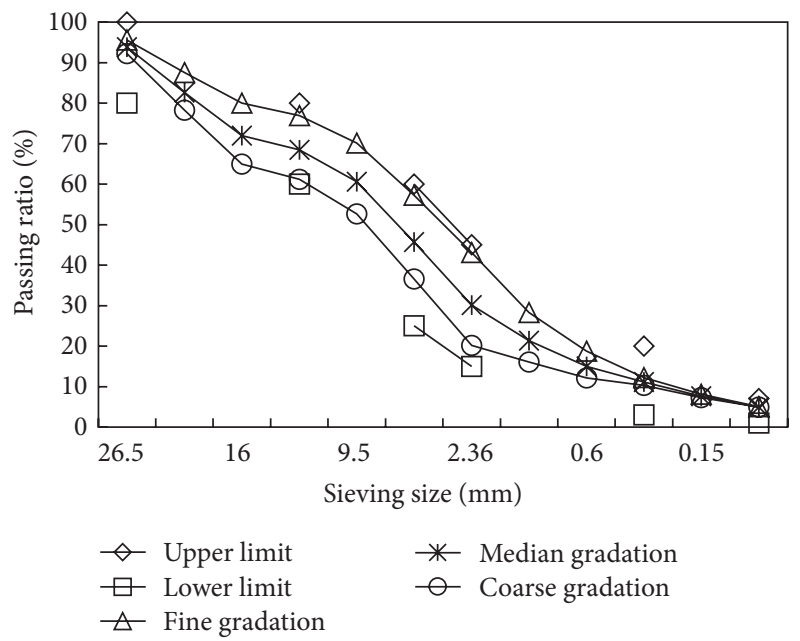

Figure 4: Different gradations used for cold recycled asphalt mixtures.

obtained with a high $R^{2}$ value. According to the linear equation, the tensile strength of pure RAP was estimated to be $0.51 \mathrm{MPa}$ when the percentage of RAP is $100 \%$. The value is within the range of 0.4 and $0.6 \mathrm{MPa}$ for tensile strength of cold recycled asphalt mixtures observed from real projects. Combined with the previous specific surface area analysis, it indicates that RAP particles are much different with real aggregates, as the main composition material in cold recycled asphalt mixture; the strength of RAP has important influences on the strength of cold recycled asphalt mixture.

3.1.2. Influence of RAP Gradation. To analyze the influences of gradation on the tensile strength of cold recycled asphalt mixtures, three different gradations named as fine gradation, median gradation, and coarse gradation were used to prepare cold recycled asphalt mixtures, as shown in Figure 4. The median gradation is the base gradation used for the controlling cold recycled asphalt mixture as described in Section 2.1. During the preparation of cold recycled asphalt mixtures, the contents of emulsified asphalt, cement, and water were $4 \%$, $1.5 \%$, and $5.4 \%$, respectively. Figure 5 summarizes the tensile 


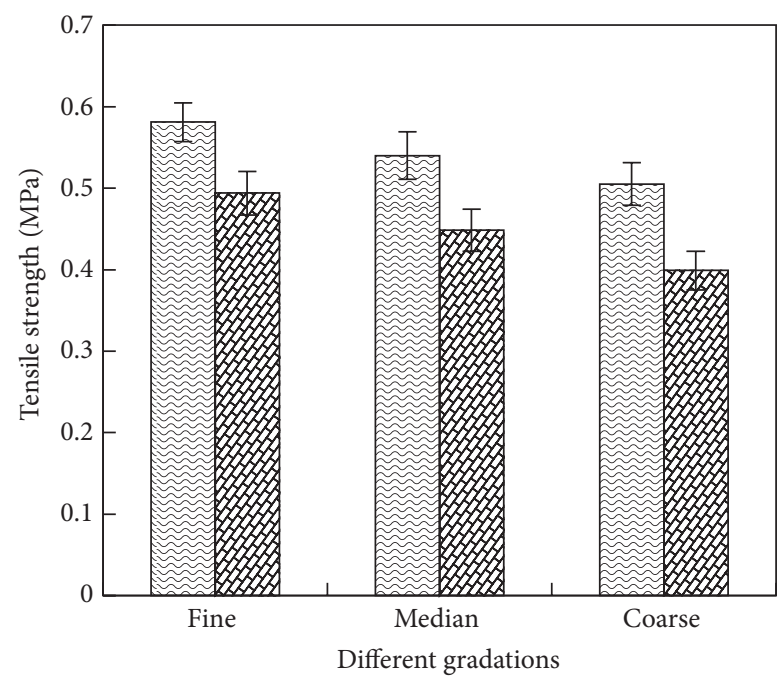

Dry splitting test

Immersion splitting test

(a) Tensile strength

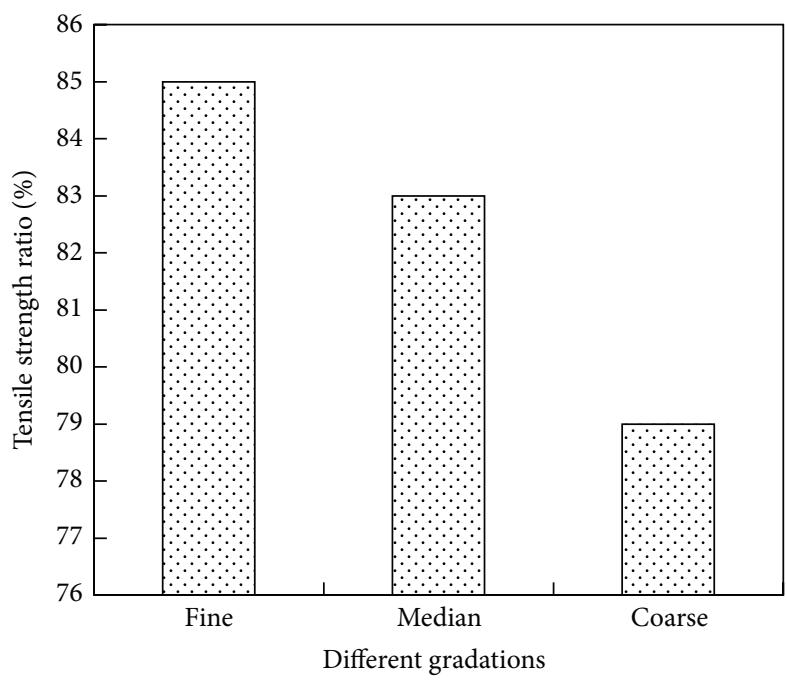

(b) Tensile strength ratio

FIGURE 5: Splitting test results for cold recycled asphalt mixtures with different gradations.

strength and tensile strength ratio from splitting tests for cold recycled asphalt mixtures with different gradations.

The results show that gradation can affect the tensile strength and moisture stability of cold recycled asphalt mixtures. Both tensile strength and tensile strength ratio of cold recycled asphalt mixtures increase as gradation becomes finer. When the fine gradation is used, there are fewer coarse RAP particles in the mixture and the specific surface area of aggregate mix increases. This could improve the bonding between new binder and RAP and thus increase the tensile strength of cold recycled asphalt mixtures. This conforms with the previous analysis of RAP.

\subsection{Effect of Emulsified Asphalt}

3.2.1. Influence of Asphalt Content. Three different emulsified asphalt contents, $3 \%, 4 \%$, and $5 \%$, were used to analyze the influence of asphalt content on the tensile strength of cold recycled asphalt mixtures. All of the three gradations described in Figure 4 were used to prepare the cold recycled asphalt mixtures. For the cold recycled asphalt mixtures with different emulsified asphalt contents of $3 \%, 4 \%$, and $5 \%$, the cement content was $1.5 \%$ and the water contents were $5.8 \%, 5.4 \%$, and 5\%, respectively. Figure 6 summarizes the tensile strength and tensile strength ratio from splitting tests for cold recycled asphalt mixtures with different gradations and asphalt contents. It is shown that, for each gradation, as the asphalt content increases from $3 \%$ to $5 \%$, both the tensile strength and tensile strength ratio increase first and then decrease. Therefore, there is optimal asphalt content to guarantee the best tensile strength and moisture stability for cold recycled asphalt mixture. For instance, the optimal asphalt content is determined to be $4 \%$ for the specimens tested in this study.
3.2.2. Influence of Emulsified Asphalt Type. Three types of emulsified asphalt, including high-viscosity slow-setting emulsified asphalt, normal slow-setting emulsified asphalt, and medium-setting emulsified asphalt, were compared in this study. The median gradation described in Figure 4 was used to prepare the cold recycled asphalt mixture. During the preparation of cold recycled asphalt mixtures, the contents of emulsified asphalt, cement, and water were $4 \%, 1.5 \%$, and $5.4 \%$, respectively. To simulate the increasing of tensile strength of cold recycled asphalt mixtures over time, samples were prepared and cured at room temperature for 2, 3, 4, and 7 days, respectively. The tensile strength by dry splitting test after different curing days is shown in Figure 7. To simulate the final tensile strength of cold recycled asphalt mixture, samples were prepared and cured at room temperature for 7 days and then at $60^{\circ} \mathrm{C}$ for another $48 \mathrm{~h}$. The final tensile strength by dry and immersion splitting tests for cold recycled asphalt mixtures with different types of emulsified asphalt is shown in Figure 8.

The results in Figure 8 show that the final tensile strengths of cold recycled asphalt mixtures with high-viscosity slowsetting emulsified asphalt and normal slow-setting emulsified asphalt are similar to each other. But Figure 7 shows that the tensile strengths of cold recycled asphalt mixture with the high-viscosity emulsified asphalt at different curing times are always greater than the tensile strength of cold recycled asphalt mixtures with low-viscosity emulsified asphalt. It means the viscosity of emulsified asphalt has no significant influence on the final tensile strength but does have influences on the early strength of cold recycled asphalt mixture which are important for traffic opening in the field. Meanwhile, the tensile strength of cold recycled asphalt mixtures with slowsetting emulsified asphalt is greater than that of cold recycled asphalt mixture with medium-setting emulsified asphalt. 

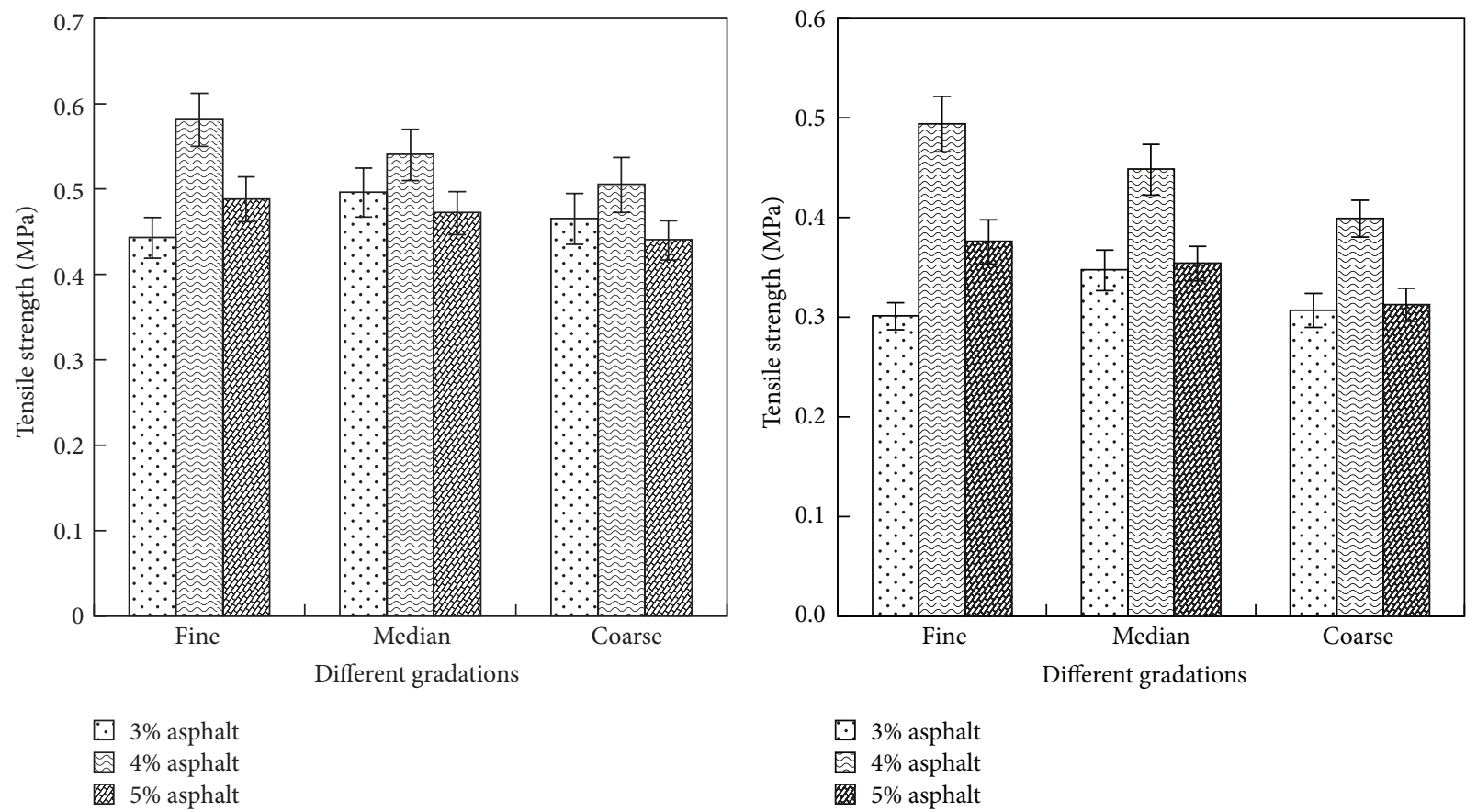

(a) Dry splitting test

(b) Immersion splitting test

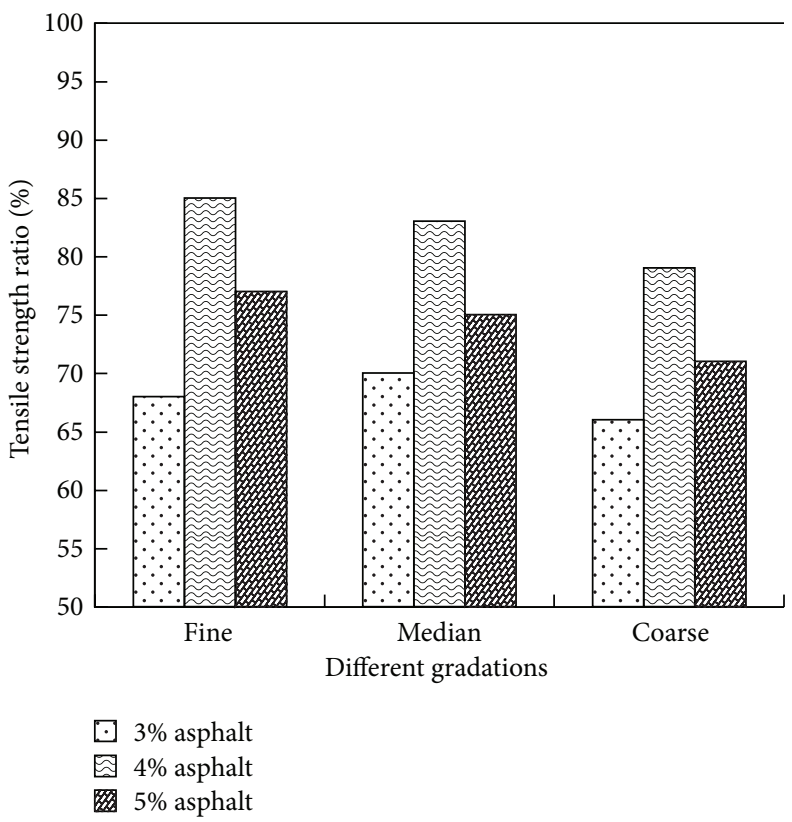

(c) Tensile strength ratio

FIGURE 6: Tensile strength of cold recycled asphalt mixtures with emulsified asphalt contents.

The amounts of water loss at different curing time for cold recycled asphalt mixtures with different types of emulsified asphalt are shown in Figure 9. It shows that the water loss of medium-setting emulsified asphalt is much greater than the other two types of slow-setting emulsified asphalt during the first three days. The greater water loss means the faster setting rate of emulsified asphalt, which can cause insufficient hydration of cement and thus the smaller strength of cold recycled asphalt mixture.

\subsection{Effect of Cement}

3.3.1. SEM Analysis of Cement-Asphalt Mix. The microstructure of cement-asphalt mix and cement hydrates after elimination of asphalt was observed using scanning electron microscope (SEM), respectively, as shown in Figures 10 and 11. Typical cement hydration products including acicular and reticular hydrated calcium silicate, columnar ettringite, and flaky calcium hydroxide can be observed in the SEM 


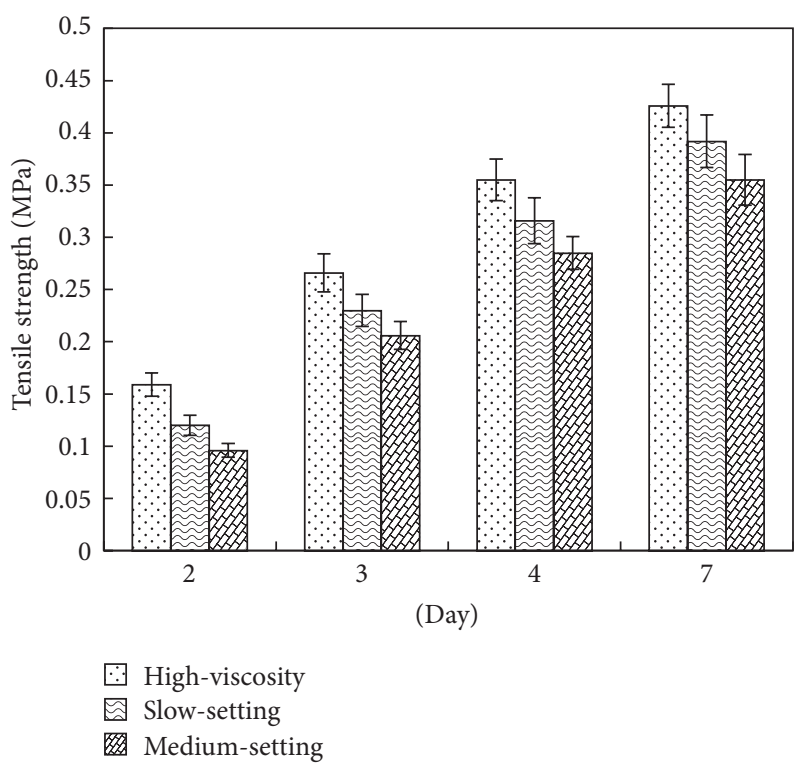

Figure 7: Tensile strength versus curing days for cold recycled asphalt mixtures with different types of emulsified asphalt.

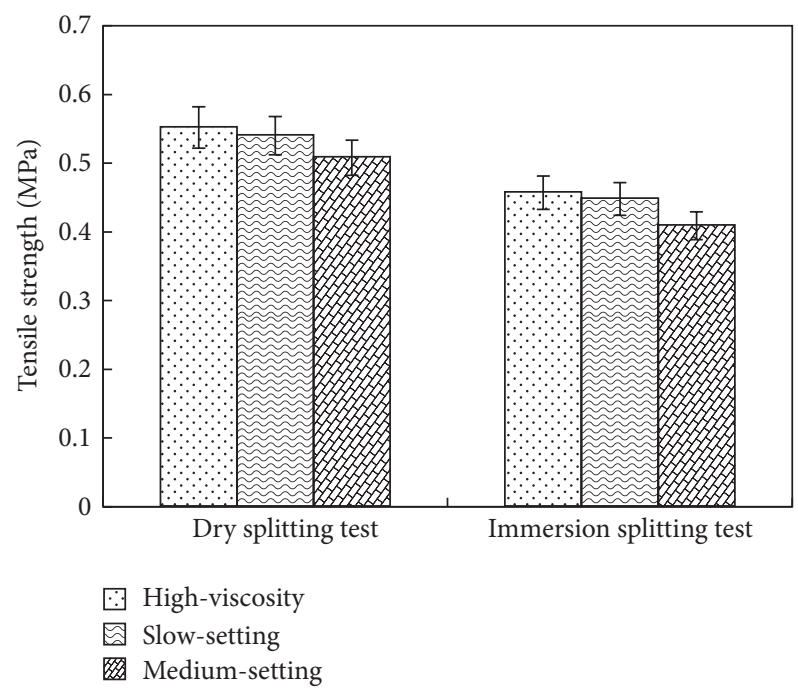

FIGURE 8: Final tensile strength for cold recycled asphalt mixtures with different types of emulsified asphalt.

images of cement-asphalt mix. It means that cement gets hydrated with water in the emulsified asphalt, which can lead to demulsification of the emulsified asphalt. The hydrated cement products interweave with asphalt to form the new binder in the cold recycled asphalt mixture. This explains the semiflexibility of the cold recycled asphalt mixture with cement hydration providing the rigidity and asphalt binder providing the flexibility [23].

However, it is observed that both asphalt and cement hydrates have disadvantageous influences on each other's microstructure integrity. As shown in Figure 10, micropores can be observed in the cement-asphalt mix that could result from water loss in the hydration reaction of cement and demulsification of emulsified asphalt. On the other hand,

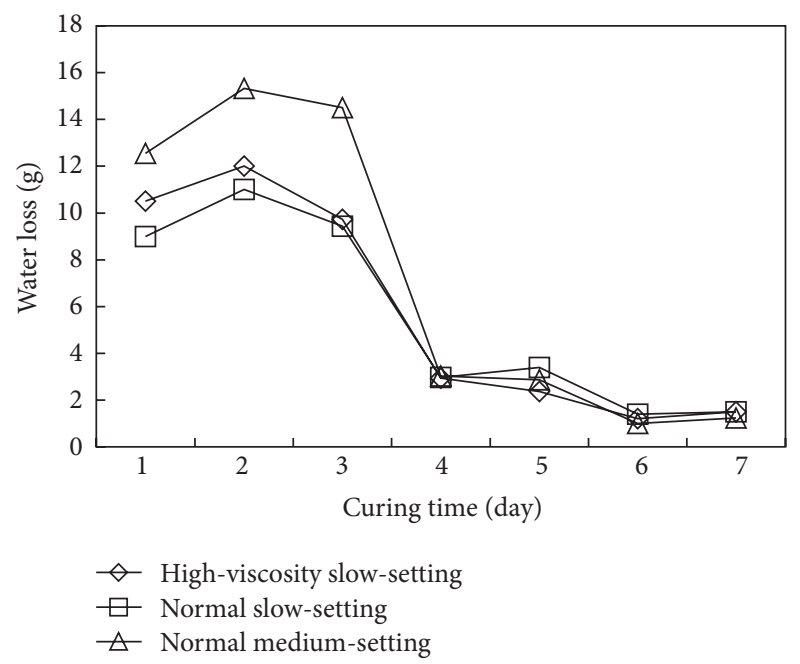

FIgURE 9: Water loss at different curing time.

a number of micropores were found in the cement hydration after elimination of asphalt, as shown in Figure 11. The cement hydrates change from massive structure into powdery form by losing its integrity and strength. Therefore, the microflaw inside the cement-asphalt mix indicates weak bonding between asphalt and hydrated cement that can cause strength defects in the cold recycled asphalt mixture. This finding is consistent with the observation reported by previous researches on the strength of cement-asphalt mortar, which indicated that asphalt membrane could have negative effects on the hydration of cement and weaken the skeleton formed by cement hydrates, while cement hydrates could impale the asphalt membrane and weaken the integrity of asphalt membrane [24].

3.3.2. Influence of Cement Content. Different cement contents, $1 \%, 1.5 \%, 2 \%, 2.5 \%$, and $3 \%$, were used to prepare testing specimens for dry and immersion splitting test. The median gradation described in Figure 4 was used to prepare the cold recycled asphalt mixture. For the cold recycled asphalt mixtures with different cement contents of $1 \%, 1.5 \%, 2 \%, 2.5 \%$, and $3 \%$, the emulsified asphalt content was $4 \%$ and the water contents were $5.2 \%, 5.4 \%, 5.4 \%, 5.6 \%$, and $5.7 \%$, respectively. Both the dry and immersion tensile strength of cold recycled asphalt mixtures with different cement contents are shown in Figure 12(a) and the tensile strength ratios at different cement contents are shown in Figure 12(b). It is clearly shown that the tensile strength increases as cement content increases. However, the relationship between the tensile strength and cement content is nonlinear. It can be seen that the increasing rate decreases when the cement content exceeds $1 \%$ and the tensile strength barely changes when the cement content exceeds $2 \%$. Compared to the tensile strength with $1.5 \%$ cement, $3 \%$ cement only improves the tensile strength by less than 10\%. Similar relationship between tensile strength ratio and cement content was observed in Figure 12(b). It can be seen that after cement content exceeds $1.5 \%$, the tensile strength ratio barely changes with the increasing of cement content. 

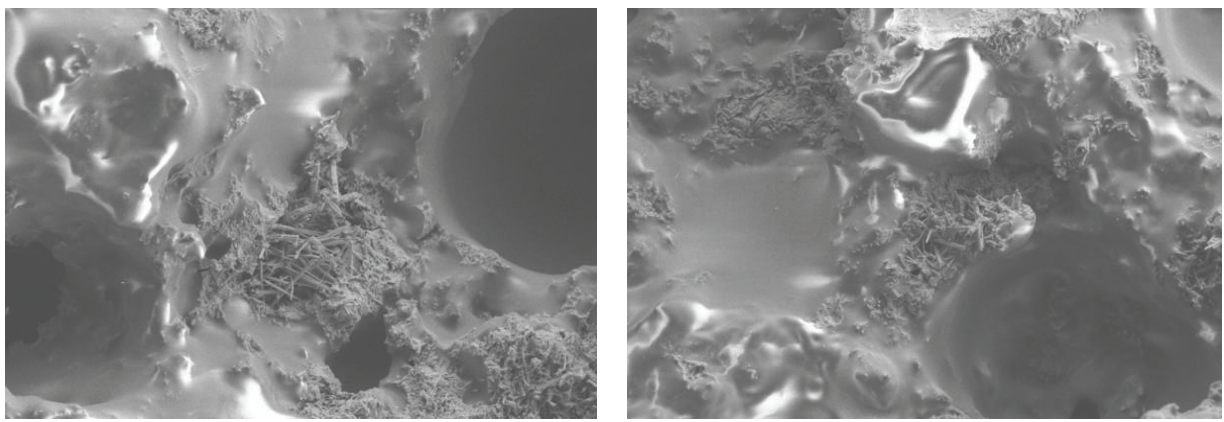

FIgURE 10: Microstructure of cement-asphalt mix.
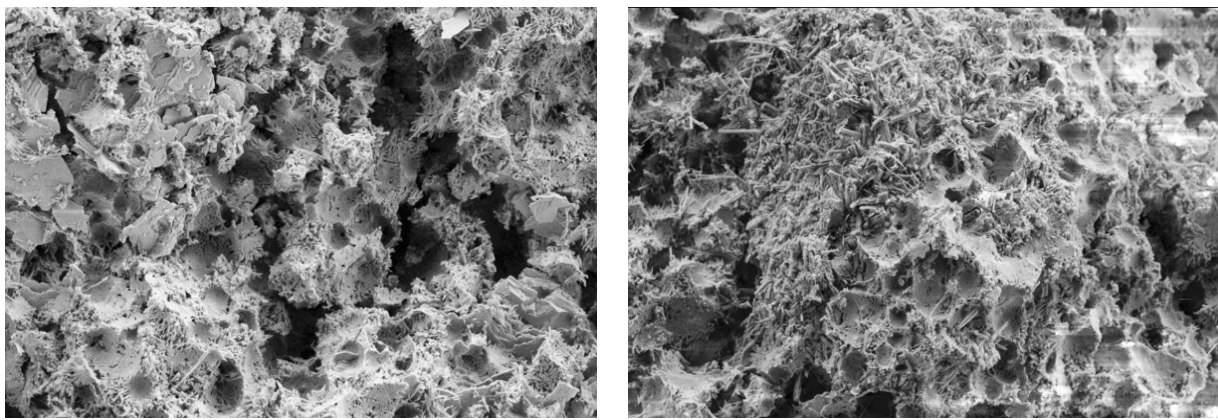

FIGURE 11: Microstructure of cement hydrates after elimination of asphalt.
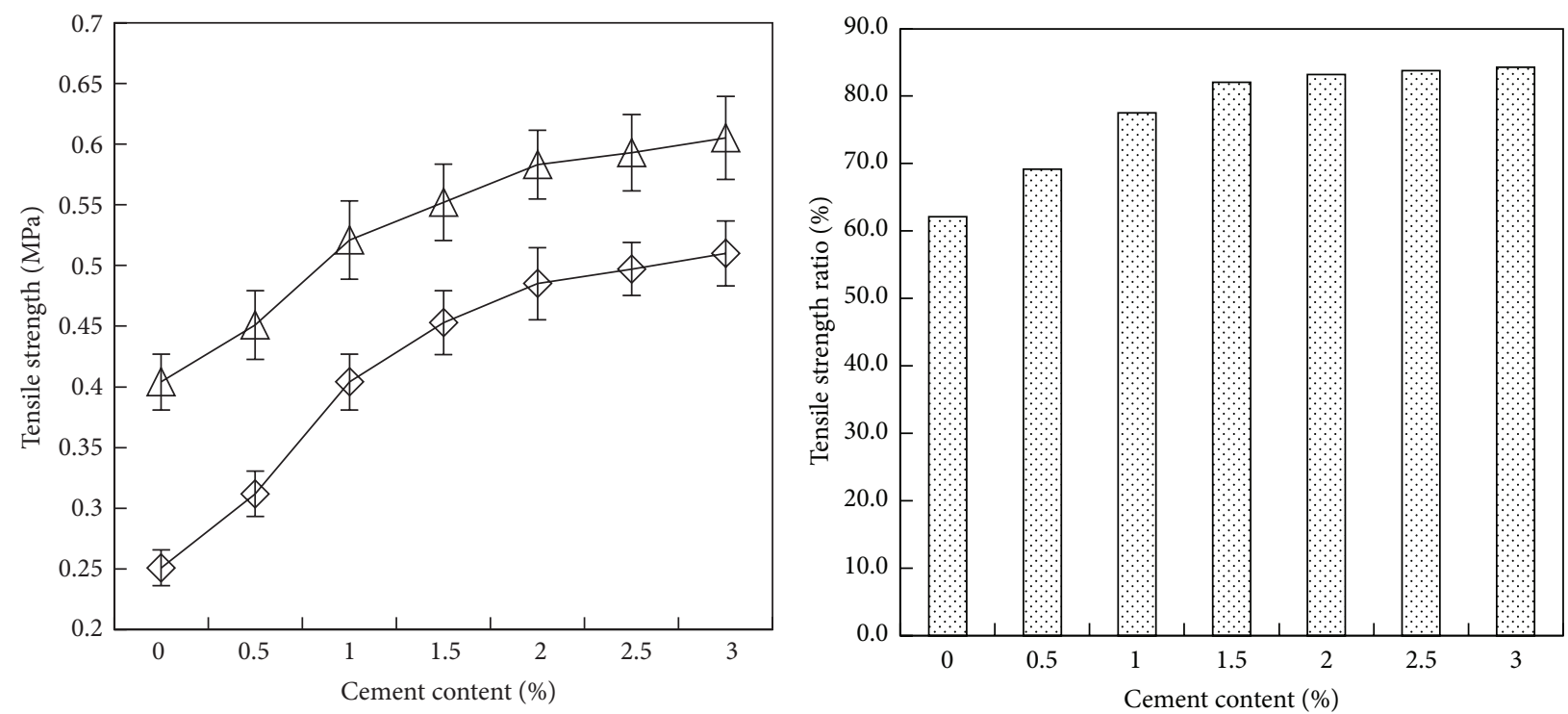

$\triangle$ Dry splitting test

$\diamond$ Immersion splitting test

(a) Dry and immersion tensile strength

(b) Tensile strength ratio

Figure 12: Tensile strength versus cement content for cold recycled asphalt mixtures.

To further confirm the effect of cement without influence of asphalt, RAP and different contents of cement were used to prepare cement-RAP mixtures without emulsified asphalt. As comparison, new aggregate and different contents of cement were used to prepare cement-aggregate mixtures. The same gradation was used for both RAP and new aggregate. Splitting tests were conducted for both cement-RAP mixtures and cement-aggregate mixtures with cement content changing from $1 \%$ to $3 \%$ by the weight of RAP or aggregate. Figure 13 summarizes the test results. 


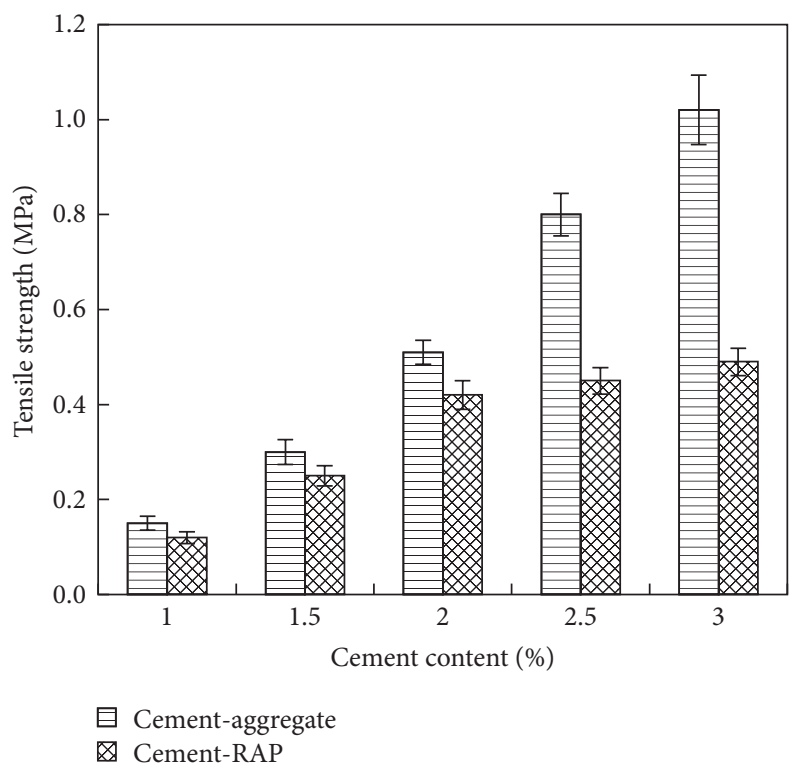

Figure 13: Tensile strength for cement-RAP mixture and cementaggregate mixture.

As shown in Figure 13, although RAP and new aggregate have the same gradation, the relationships between the tensile strength and the cement content are much different for cement-RAP mixture and cement-aggregate mixture. It is clearly indicated that the tensile strength of the cementaggregate mixture keeps growing linearly as the cement content increases. When the cement content is smaller than $2 \%$, the tensile strength of the cement-RAP mixture grows linearly with the increasing of cement content. After the cement content exceeds $2 \%$, the tensile strength of the cement-RAP mixture barely grows with the increase of cement content. The cement-RAP mixture has obviously smaller tensile strength compared to the cement-aggregate mixture with the same gradation and cement content. The tensile strength of the cement-RAP mixture with $3 \%$ cement is about $0.5 \mathrm{MPa}$ which is only half of the tensile strength of the cement-aggregate mixture with $3 \%$ cement. Combined with previous analysis in Section 3.1, it proves that the strength of RAP has decisive influence on the tensile strength of mixtures and it can limit the effect of cement in cold recycled asphalt mixture.

\section{Conclusions}

Based on laboratory testing of cold recycled asphalt mixtures, the following conclusions can be made:

(1) RAP cannot be simply treated as black aggregate in the cold recycled asphalt mixture. The specific surface area of RAP is much smaller than the aggregate in RAP that affects the bonding between RAP and new binder. Adequate crushing of RAP, addition of new aggregates, and fine gradation design can increase the tensile strength of cold recycled asphalt mixture by reducing agglomeration of RAP and improving the bonding between RAP and binder.
(2) The optimum emulsified asphalt content can be determined to achieve the highest tensile strength of cold recycled asphalt mixtures. It is suggested that slowsetting emulsified asphalt can provide enough time for cement to hydrate which is beneficial for the final strength of the cold recycled asphalt mixture. The viscosity of emulsified asphalt has no significant influences on the final tensile strength but it does affect the early strength of the cold recycled asphalt mixture. The higher viscosity of emulsified asphalt can provide better early strength of the cold recycled asphalt mixture that is helpful to carry traffic loading during initial period of traffic opening.

(3) Cement can improve the tensile strength of cold recycled asphalt mixtures by providing cement hydrates and promote demulsification of emulsified asphalt. However, microstructure flaws were observed in the mix of asphalt and cement hydration products, which could reduce the bonding between asphalt and cement and the strength of cement hydrates. Meanwhile, the influences of cement and its content on the strength of cold recycled asphalt mixture are not as significant as that on the strength of cement concrete. The effect of cement is limited by the structure and strength of RAP.

(4) It is found that factors related to emulsified asphalt, cement, and gradation design can affect the tensile strength of cold recycled asphalt mixture. But the inner structure and tensile strength of RAP have decisive influence on the tensile strength of cold recycled asphalt mixture.

\section{Conflict of Interests}

The authors declare that there is no conflict of interests regarding the publication of this paper.

\section{Acknowledgments}

The study is financially supported by the National Natural Science Foundation of China (no. 51378006), National Science and Technology Support Program (2014BAG05B04), Huoyingdong Foundation of the Ministry of Education of China (no. 141076), Excellent Young Teacher Program of Southeast University (2242015R30027), and State Key Laboratory of High Performance Civil Engineering Materials.

\section{References}

[1] F. P. Xiao, S. N. Amirkhanian, B. J. Putman, and H. Juang, "Feasibility of Superpave gyratory compaction of rubberized asphalt concrete mixtures containing reclaimed asphalt pavement," Construction and Building Materials, vol. 27, no. 1, pp. 432-438, 2012.

[2] F. Hong, D.-H. Chen, and M. M. Mikhail, "Long-term performance evaluation of recycled asphalt pavement results from 
Texas: pavement studies category 5 sections from the longterm pavement performance program," Transportation Research Record, vol. 2180, pp. 58-66, 2010.

[3] H. Wen, J. Lu, and T. M. VanReken, "Modeling the effects on energy and carbon dioxide from the use of recycled asphalt pavement in hot mix asphalt," International Journal of Sustainable Transportation, vol. 8, no. 4, pp. 249-261, 2014.

[4] X. Yu and Y. Li, "Optimal percentage of reclaimed asphalt pavement in central plant hot recycling mixture," Journal of Wuhan University of Technology-Mater. Sci. Ed., vol. 25, no. 4, pp. 659-662, 2010.

[5] C. Plati, A. Loizos, V. Papavasiliou, and A. Kaltsounis, "Investigating in situ properties of recycled asphalt pavement with foamed asphalt as base stabilizer," Advances in Civil Engineering, vol. 2010, Article ID 565924, 10 pages, 2010.

[6] G. Thenoux, Á. González, and R. Dowling, "Energy consumption comparison for different asphalt pavements rehabilitation techniques used in Chile," Resources, Conservation and Recycling, vol. 49, no. 4, pp. 325-339, 2007.

[7] Wirtgen Cold Recycling Manual, Wirtgen GmbH, Windhagen, Germany, 2010.

[8] J. H. Yan, H. R. Zhu, Z. X. Zhang, L. Gao, and S. Charmot, "The theoretical analysis of the RAP aged asphalt influence on the performance of asphalt emulsion cold recycled mixes," Construction and Building Materials, vol. 71, pp. 444-450, 2014.

[9] Y. Kim and H. D. Lee, "Performance evaluation of Cold In-Place Recycling mixtures using emulsified asphalt based on dynamic modulus, flow number, flow time, and raveling loss," KSCE Journal of Civil Engineering, vol. 16, no. 4, pp. 586-593, 2012.

[10] S. Charmot and P. Romero, "Assessment of fracture parameters to predict field cracking performance of cold in-place recycling mixtures," Transportation Research Record, vol. 2155, pp. 34-42, 2010.

[11] F. Long and H. Theyse, "Mechanistic-empirical structural design models for foamed and emulsified bitumen treated materials," in Proceedings of the 8th Conference on Asphalt Pavements for Southern Africa (CAPSA '04), Sun City, South Africa, September 2004.

[12] T. S. Ozsahin and S. Oruc, "Neural network model for resilient modulus of emulsified asphalt mixtures," Construction and Building Materials, vol. 22, no. 7, pp. 1436-1445, 2008.

[13] J. H. Yan and Z. X. Zhang, "The correlativity analysis between the aggregates characteristics of reclaimed asphalt pavement and the performance of emulsion cold recycling mixture," in Proceedings of the International Symposium on Heavy Duty Asphalt Pavements and Bridge Deck Pavements, Nanjing, China, May 2012.

[14] R. Issa, M. M. Zaman, G. A. Miller, and L. J. Senkowski, "Characteristics of cold processed asphalt millings and cementemulsion mix," Transportation Research Record, vol. 1767, pp. 16, 2001.

[15] B. Gómez-Meijide and I. Pérez, "A proposed methodology for the global study of the mechanical properties of cold asphalt mixtures," Materials and Design, vol. 57, pp. 520-527, 2014.

[16] M. A. T. Alsheyab and T. S. Khedaywi, "Effect of electric arc furnace dust (EAFD) on properties of asphalt cement mixture," Resources, Conservation and Recycling, vol. 70, pp. 38-43, 2013.

[17] A. Modarres, M. Rahimzadeh, and M. Zarrabi, "Field investigation of pavement rehabilitation utilizing cold in-place recycling," Resources, Conservation and Recycling, vol. 83, pp. 112120, 2014.
[18] A. Forsberg, E. Lukanen, and T. Thomas, "Engineered cold inplace recycling project: Blue Earth County State Aid Highway 20, Minnesota," Transportation Research Record, vol. 1813, pp. 111-123, 2002.

[19] Y. Kim, S. Im, and H. D. Lee, "Impacts of curing time and moisture content on engineering properties of cold in-place recycling mixtures using foamed or emulsified asphalt," Journal of Materials in Civil Engineering, vol. 23, no. 5, pp. 542-553, 2011.

[20] E. S. Peter, B. Gabriel, H. Edgard, D. Weitzel, and S. Bemanian, "Performance of cold in-place recycling in Nevada," Transport Research Record, vol. 1896, pp. 162-169, 2007.

[21] Ministry of Transport of the People's Republic of China, Technical Specification for Construction of Highway Asphalt Pavement, 2006.

[22] Ministry of Transport of the People's Republic of China, Technical Specification for Highway Asphalt Pavement Recycling, 2008.

[23] L. He, Research on constructing semi-flexible pavement by cold in-place recycling of asphalt pavement [Ph.D. thesis], Chongqing Jiaotong University, Nan'an, China, 2008.

[24] W. Qiang, Y. Peiyu, A. Ruhan, Y. Jinbo, and K. Xiangming, "Strength mechanism of cement-asphalt mortar," Journal of Materials in Civil Engineering, vol. 23, no. 9, pp. 1353-1359, 2011. 

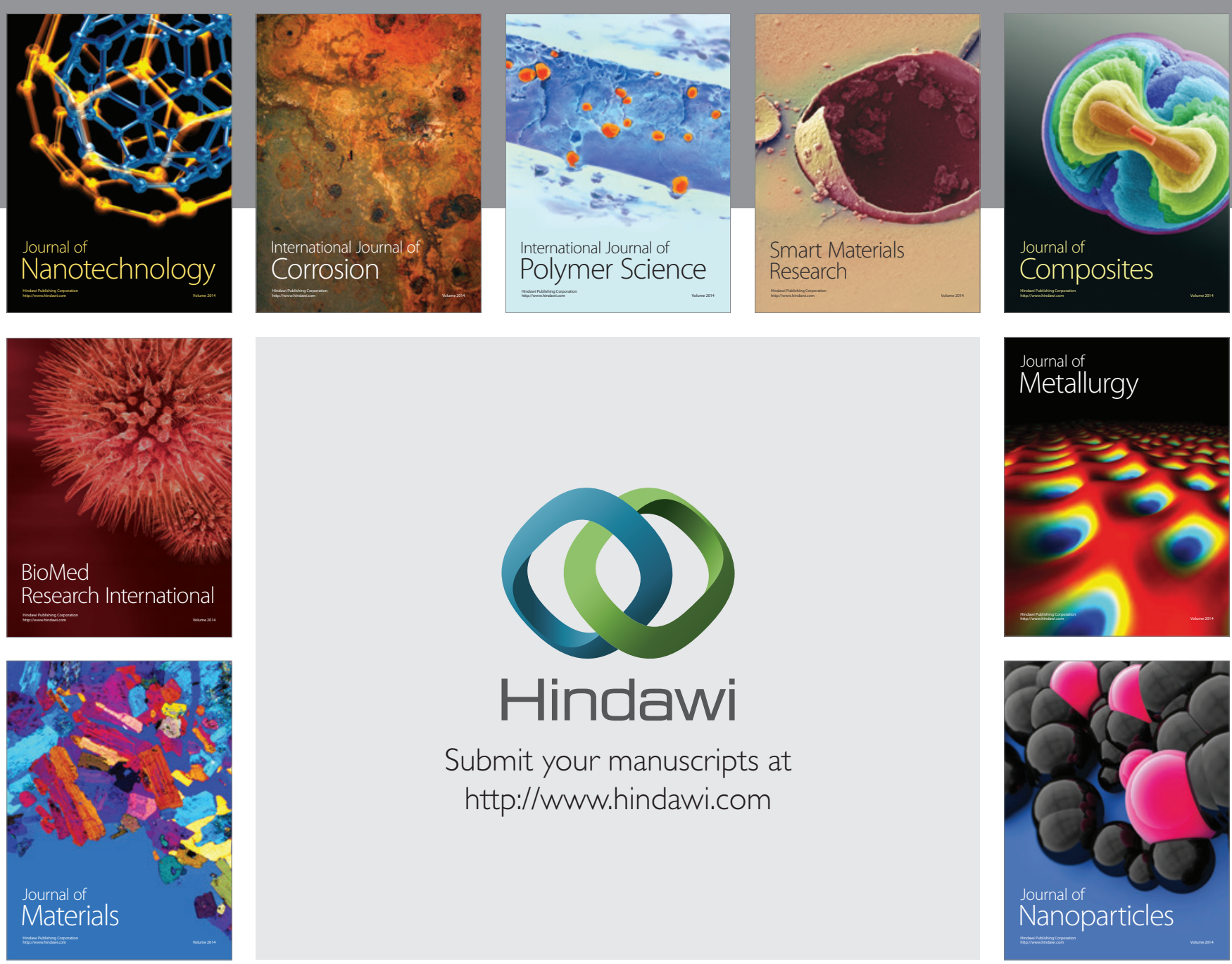

Submit your manuscripts at http://www.hindawi.com
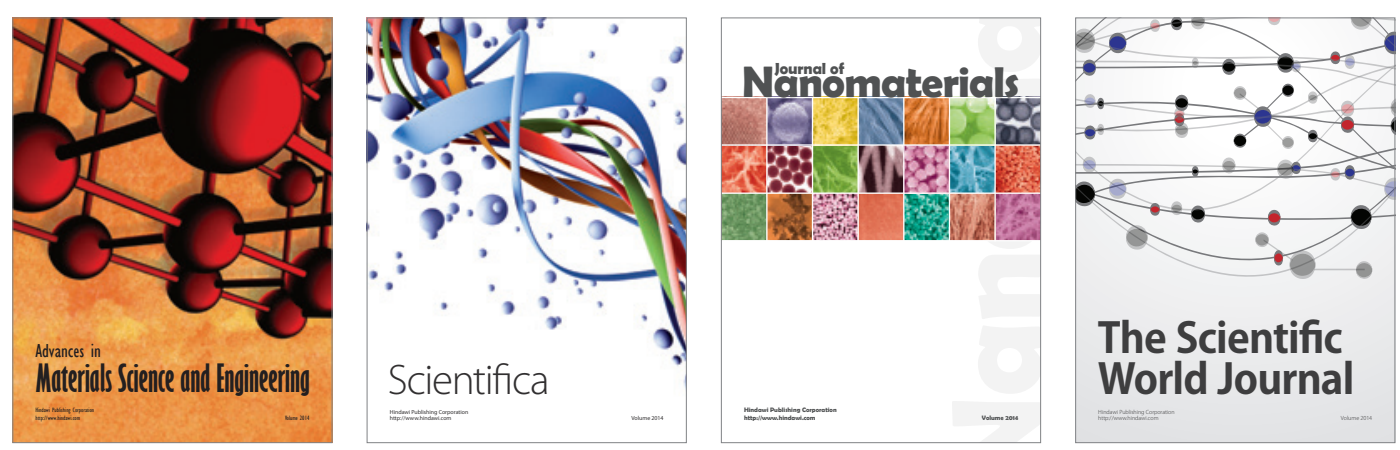

\section{The Scientific World Journal}
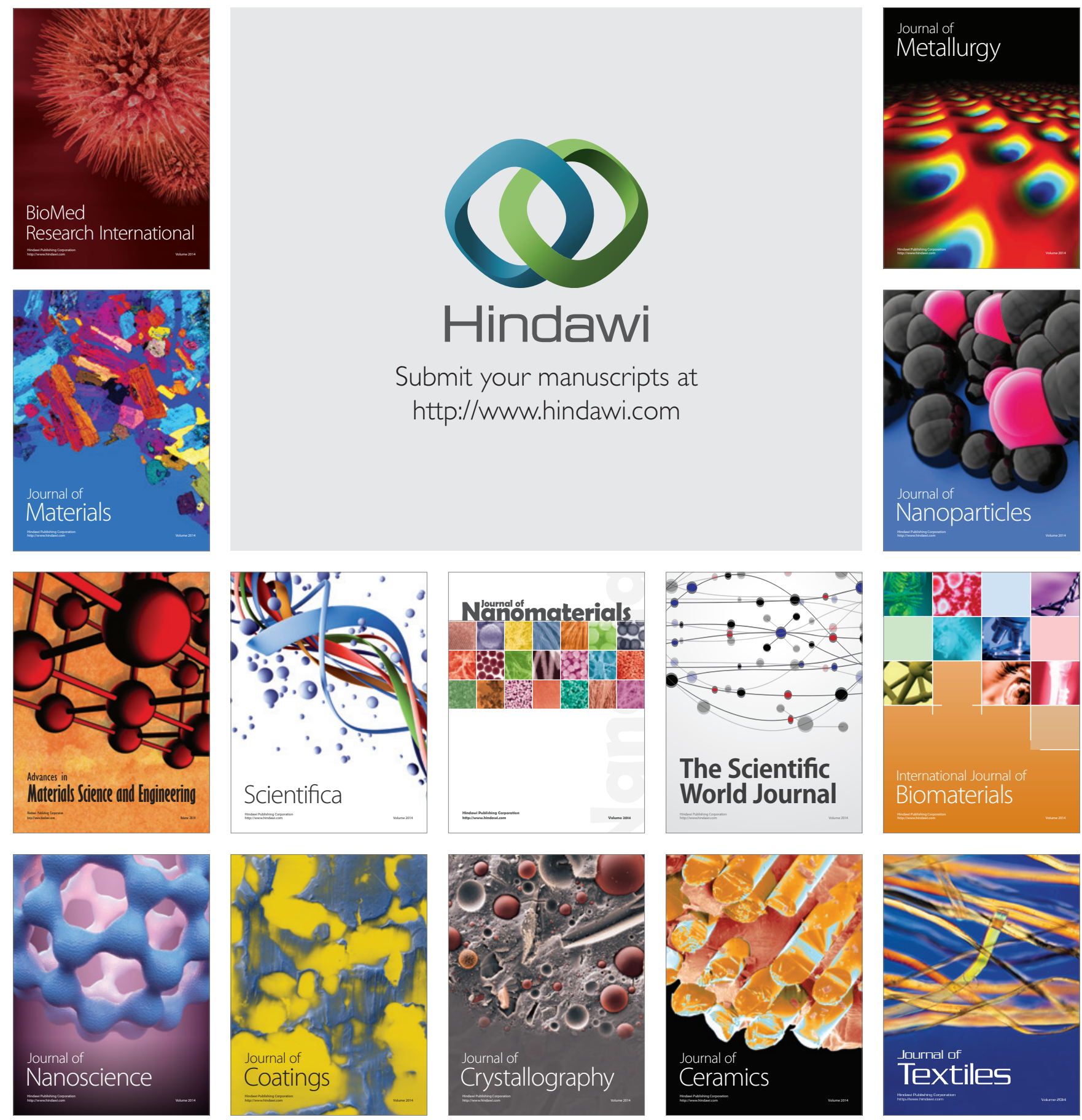